$6 \quad{ }^{1}$ Molecular Parasitology Laboratory, Immunology Department, QIMR Berghofer Medical

7 Research Institute, Brisbane, Australia

$8 \quad{ }^{2}$ Cancer Drug Mechanisms Group, Cell \& Molecular Biology Department, QIMR Berghofer

9 Medical Research Institute, Brisbane, Australia

${ }^{3}$ Scabies Group, Cell \& Molecular Biology Department, QIMR Berghofer Medical Research

\section{Kunitz type protease inhibitor EgKI-1 from the canine tapeworm Echinococcus granulosus as a promising anti-cancer therapeutic}

\section{Shiwanthi L Ranasinghe ${ }^{1^{*}}$, Glen M Boyle ${ }^{2}$, Katja Fischer ${ }^{3}$, Jeremy Potriquet ${ }^{4}$, Jason P Mulvenna $^{5}$, Donald P McManus ${ }^{1}$}

Institute, Brisbane, Australia

${ }^{4}$ Australian Institute of Tropical Health \& Medicine, James Cook University, Cairns, Australia

${ }^{5}$ Biomarkers and Biology of Infection Related Cancers Laboratory, QIMR Berghofer Medical Research Institute, Brisbane, Australia

*Corresponding author - shiwanthi.ranasinghe@,qimrberghofer.edu.au 


\section{Abstract}

EgKI-1, a member of the Kunitz type protease inhibitor family, is highly expressed by the oncosphere of the canine tapeworm Echinococcus granulosus, the stage that is infectious to humans and ungulates, giving rise to a hydatid cyst localized to the liver and other organs. Larval protoscoleces, which develop within the hydatid cyst, have been shown to possess anti-cancer properties, although the precise molecules involved have not been identified. We show that recombinant EgKI-1 inhibits the growth and migration of a range of human cancers including breast, melanoma and cervical cancer cell lines in a dose-dependent manner in vitro without affecting normal cell growth. Furthermore, EgKI-1 treatment arrested the cancer cell growth by disrupting the cell cycle and induced apoptosis of cancer cells in vitro. An in vivo model of triple negative breast cancer (MDA-MB-231) in BALB/c nude mice showed significant tumor growth reduction in EgKI-1-treated mice compared with controls. These findings indicate that EgKI-1 shows promise for future development as an anti-cancer therapeutic.

\section{Introduction}

Protein-based therapeutics enable targeted approaches for treating cancer (1). There are many benefits of proteins over small-molecule drugs mainly because of the increased surface area accessing a much wider range of protein targets (2). Protease inhibitors are important as potential cancer therapeutics as proteases are associated with carcinogenesis and cancer progression. Numerous plant protease inhibitors have recently entered human clinical trials (3). Parasites produce a range of protease inhibitors with diverse functions mainly to evade hostile adverse host reactions (4).

Several parasites, including the liver flukes, Opisthorchis viverrini and Clonorchis sinensis, and the blood fluke, Schistosoma haematobium, are known risk factors for cholangiocarcinoma and bladder cancer, respectively (5). In contrast, other parasites such as Trypanosoma cruzi (6), Toxoplasma gondii (7) and Echinococcus granulosus (8) produce metabolites with anticancer properties.

An increasing number of studies have shown that the presence of neutrophils in tumors, known as tumor associated neutrophils (TAN) correlates with poor prognosis (9), specifically in breast cancers (10). Neutrophils play major roles in tumor initiation, growth and metastasis (11) mainly through the serine protease enzyme neutrophil elastase secreted by active 
neutrophils. Neutrophil elastase also acts as a chemoattractant for more neutrophils (12). Therefore, potent neutrophil elastase inhibitors have stimulated much interest for development as cancer therapeutics (13).

The larval stage of the canine tapeworm (phylum Cestoda) E. granulosus causes echinococcosis (hydatidosis) in humans and ungulates (sheep, goats, cattle etc) when they ingest the parasite eggs containing oncospheres in contaminated food or water (14). The oncospheres hatch and penetrate the intestinal mucosa, enter the blood stream and migrate to the liver or lung. A fluid-filled larva begins to develop from a single oncosphere with subsequent formation of multiple layers, resulting in a metacestode or hydatid cyst (15). Protoscoleces, which develop asexually within the hydatid cyst, have been shown to induce the death of fibrosarcoma cells although the specific molecules involved have not been identified (8). We have shown that EgKI-1, a member of the Kunitz type protease inhibitor family, is highly expressed in oncospheres, is a potent neutrophil elastase and chemotaxis inhibitor (16) and was recently granted an International Patent Publication (17).

In this study, recombinant EgKI-1 was expressed in yeast, purified and investigated for potential anti-cancer properties in vitro and in vivo.

\section{Methods}

\section{EgKI-1 expression in yeast}

The pPICZ $\alpha$ A plasmid containing the EgKI-1 gene sequence and EcoRI/ XbaI cloning sites was synthesized by Biomatik (Wilmington, USA). The plasmid (10 ng) was then transformed into E. coli XL1-Blue competent cells (Stratagene, San Diego, USA) and sequenced to confirm the integrity of the insertion. Vector bearing the confirmed sequence was inserted into Pichia pastoris $\mathrm{KM} 71 \mathrm{H}$ cells using the electroporation method described by Invitrogen $^{\mathrm{TM}}$ (Carlsbad, USA). Briefly, a single colony of XL1-Blue cells, bearing the confirmed EgKI-1 sequence isolated from a low salt LB agar plate, was grown in $5 \mathrm{ml}$ of low salt LB medium. From these cells, DNA was extracted using a Plasmid Midi kit (Qiagen, Hilden, Germany). DNA was linearized with SacI-HF (New England BioLabs, Ipswich, USA), extracted using phenol/chloroform and re-suspended in $10 \mathrm{mM}$ Tris ( $\mathrm{pH} \mathrm{8.5)} \mathrm{buffer.}$ Linearized DNA $(25 \mu \mathrm{g})$ was then mixed with $80 \mu \mathrm{KM}$ K11 H cells on ice and transferred to a $0.2 \mathrm{~cm}$ cuvette and an electric shock applied using Gene Pulser (Biorad, Hercules, USA). Then $1 \mathrm{ml}$ of $1 \mathrm{M}$ sorbitol $+200 \mu \mathrm{l}$ HEPES mixture was added to the cells and transferred to 
a $10 \mathrm{ml}$ tube. Cells were then incubated for 1.5 hours at $30^{\circ} \mathrm{C}$, plated on YPD agar supplemented with $100 \mu \mathrm{g} / \mathrm{ml}$ zeocin, and incubated at $30^{\circ} \mathrm{C}$ for 2-3 days. A single colony from the YPD plate was then picked and inoculated into BMGY complete medium (50 ml) and incubated at $30^{\circ} \mathrm{C}$ with $200 \mathrm{rpm}$ agitation for 24 hours. Frozen stocks were then made with $100 \%$ sterilized glycerol and stored at $-80^{\circ} \mathrm{C}$ for future use. The remainder of the starter culture was then used to inoculate $1 \mathrm{~L}$ of BMGY complete medium and grown with $200 \mathrm{rpm}$ agitation for 24 hours at $30^{\circ} \mathrm{C}$. On the following day, cells were harvested by centrifuging at $4000 \mathrm{rpm}$ for $10 \mathrm{~min}$ at room temperature and re-suspended in $200 \mathrm{ml} \mathrm{YNB}$ media. Cells were grown with $200 \mathrm{rpm}$ agitation for 96 hours at $30^{\circ} \mathrm{C}$ and then induced with $100 \%$ methanol to a final concentration of $0.5 \%$ every 24 hours to induce expression of the EgKI-1 gene under the AOX1 promoter.

\section{Protein purification and identification}

The cultured supernatant $(200 \mathrm{ml})$ was then collected by centrifugation at $10,000 \mathrm{rpm}$ for 30 min at $4{ }^{\circ} \mathrm{C}$ and stored at $-80{ }^{\circ} \mathrm{C}$ until required. The supernatant was thawed, dialyzed into 50 $\mathrm{mM}$ MES buffer (pH 6) and filtered through a $0.45 \mu \mathrm{m}$ filter before being loaded on to a Hitrap SP sepharose column (GE Healthcare Life Sciences) pre-equilibrated with $50 \mathrm{mM}$ MES buffer ( $\mathrm{pH}$ 6) (18). Unbound material was removed by washing with equilibration buffer and protein was eluted using a linear gradient of $0-1 \mathrm{M} \mathrm{NaCl}$ over $40 \mathrm{ml}$, with EgKI-1 eluting between 0.4 and $0.6 \mathrm{M} \mathrm{NaCl}$. Purification was monitored by analysis on SDS-PAGE gels and protease inhibitory activity in fractions containing EgKI-1. Purified EgKI-1 was then dialyzed into $50 \mathrm{mM}$ Tris $120 \mathrm{mM} \mathrm{NaCl}$ (pH 7) buffer and quantified using the Bradford protein assay (19). A sample of the EgKI-1 protein was visualized on 15\% SDS-PAGE to verify its purity and the EgKI-1 gel band was subjected to in-gel trypsin digestion and nano high performance liquid chromatography coupled to mass spectrometry (nano LC-MS) (20).

\section{Cell lines}

Human cancer cell lines including breast adenocarcinoma (MCF-7), breast ductal carcinoma (T47D), mammary gland epithelial adenocarcinoma (MDA-MB-231), pharynx squamous epithelial carcinoma $(\mathrm{FaDu})$, cervical epithelial adenocarcinoma $(\mathrm{HeLa})$, tongue squamous cell carcinoma (SCC15) and melanoma (CJM) were used to determine the effect of EgKI-1 on cell growth. Primary neonatal foreskin fibroblast (NFF) cells were used as normal human cell controls. All cells were cultured in complete media (RPMI-1640 supplemented with 10\% 
(v/v) heat-inactivated fetal calf serum (Thermo Fisher Scientific, Waltham, USA), 3 mM 4(2-hydroxyethyl)-1-piperazineethanesulfonic acid (HEPES)) and $100 \mathrm{U} / \mathrm{ml}$ penicillin and 100 $\mu \mathrm{g} / \mathrm{ml}$ streptomycin (Thermo Fisher Scientific). Cell line identity was checked by Short Tandem Repeat (STR) profiling with the GenePrint ${ }^{\circledR} 10$ system (Promega, Madison, WI) according to manufacturer's instructions. Cultured cells were routinely checked for mycoplasma infection by a specific PCR-based assay (21) and were always negative.

\section{Cell growth assays}

Cells were seeded at 5000 cells per well in 96-well plates and incubated overnight at $37^{\circ} \mathrm{C}$. On the following day cells were treated with different concentrations of the EgKI-1 protein and control wells with buffer alone and cultured for 3-5 days until the control non-treated wells were 95\% confluent. Cell lines were then assayed using sulforhodamine B (SRB) to measure the inhibition of cell growth (22). The concentration of EgKI-1 needed to inhibit cell growth by $50 \%\left(\mathrm{IC}_{50}\right)$ was calculated using GraphPad Prism 7 software. The experiments were repeated three times and the mean \pm SEM was determined. Growth of cancer cells was also monitored real time with the IncuCyte Zoom system in the presence of varying concentrations of EgKI-1.

\section{Cell migration analysis}

In vitro scratch wound assays were carried out to determine the effect of EgKI-1 on cancer cell migration (23). This method mimics, to some extent, the migration of cells in vivo and is more informative and convenient than other methods(23). CJM, MDA-MB-231 and HeLa cells were grown to create a confluent monolayer in 96-well plates. Then the monolayers were scraped in a straight line to create a "scratch" using the Wound Maker-IncuCyte ZOOM-Image Lock Plate system and washed once with growth medium. After replacing the medium, cells were treated with $0.5 \mu \mathrm{M}$ EgKI-1 or control buffer and monitored by IncuCyte Zoom. Images were acquired for each well at 12 hours interval by an in built phase contrast microscope.

The distance between one side of scratch and the other at each time point was measured using IncuCyte Zoom software. The Wound width $(\mu \mathrm{m})$ obtained at different time points was then statistically analyzed with 2-way ANOVA using GraphPad Prism version 7. 


\section{Immunocytochemistry}

Cells were grown in 8-well tissue culture chambers on slides (Sarstedt, Nümbrecht, Germany) overnight. On the following day, different chambers were treated with EgKI-1 (2 $\mu \mathrm{M})$ and control buffer. After 24 hours incubation, supernatants were removed and cells were fixed in ice cold methanol for 10 min followed by two washes with PBS. Cells were then permeabilized with ethanol: acetic acid $(2: 1)$ at $-20^{\circ} \mathrm{C}$ for $5 \mathrm{~min}$. After $3 \times 1 \mathrm{~min}$ rinses in PBS, normal donkey serum (10\%) was applied for $20 \mathrm{~min}$ at room temperature (RT). Excess normal serum was decanted and mouse anti-EgKI-1 antibody (16) and rabbit anti-tubulin antibody (Abcam, Cambridge, UK) diluted 1:80 in PBS were applied for 60 min at RT. Cells were washed with three changes of PBS and Alexa fluor donkey anti-mouse 488 and Alexa fluor donkey anti-rabbit 555 diluted 1:200 in PBS were applied for $30 \mathrm{~min}$ at RT. After washing with PBS, cells were stained with DAPI (1:35000 in PBS) for 2-5 min followed by another PBS wash. Prolong fluorescence mount (Thermo Fisher Scientific) was then applied to each slide and a coverslip applied. Cells were visualized under a Zeiss 780-NLO point scanning confocal microscope for the presence of EgKI-1 and tubulin. Fluorescence intensity of tubulin and total cell areas were then measured with ImageJ software (24) and statistically analyzed with GraphPad Prism version 7.

\section{Cell cycle phase analysis}

Cells were analyzed using propidium iodide (PI) staining according to a published protocol (25). Briefly, MDA-MB-231 and HeLa cells were treated with $1 \mu \mathrm{M}$ EgKI-1 or control buffer and cells were harvested at 24 and 48 hours after treatment. At both time points, cells in the control and EgKI-1 treated wells were collected in to $5 \mathrm{ml}$ polystyrene round-bottom tubes by trypsinisation and washed thoroughly with PBS. The cells were then fixed with ice-cold $70 \%$ ethanol and stored at $4{ }^{\circ} \mathrm{C}$ overnight. The ethanol was discarded after centrifugation and the cells were washed with PBS. Cells were incubated with PI/triton X-100 solution $(0.02 \% \mathrm{w} / \mathrm{v}$ DNase free RNase, $2 \% \mathrm{w} / \mathrm{v}$ PI in $0.1 \% \mathrm{v} / \mathrm{v}$ triton X-100) for $30 \mathrm{~min}$ at RT and analyzed with a LSR Fortessa flow cytometer using a YG610/20 filter. ModFit LT software was used to complete the cell cycle analysis.

\section{Analysis of apoptosis}

An Annexin V-FITC apoptosis detection kit (Biotool, York, UK) was used to determine whether EgKI-1 induced apoptosis in cancer cells. Cells were seeded at $\sim 60 \%$ confluency in 
24-well plates and EgKI-1 or control buffer treatment was carried out on the following day. After 24 hours incubation, cells were harvested by trypsinisation and washed with cold PBS. Equal amounts of Annexin V-FITC/ PI were added to the cells re-suspended in 1x binding buffer (10 mM HEPES (pH 7.4), $140 \mathrm{mM} \mathrm{NaCl}, 2.5 \mathrm{mM} \mathrm{CaCl}_{2}$ ). Cells were then incubated at room temperature for 15 min and analyzed with a BD LSR Fortessa flow cytometer using a YG610/20 filter for PI and a B530/30 filter for FITC. FlowJo version 10 was used to analyze cell staining and to determine the percentage positivity. Annexin $\mathrm{V}^{+} / \mathrm{PI}^{-}$cells were considered as early apoptotic cells and Annexin $\mathrm{V}^{+} / \mathrm{PI}^{+}$cells were considered as late apoptotic cells.

\section{Proteomics quantitative analysis following EgKI-1 exposure}

To determine which proteins in MDA-MB-231 cancer cells were affected by EgKI-1 treatment, quantitative SWATH-MS (sequential window acquisition of all theoretical spectramass spectrometry) was used as described by Gill et al(26) was used with few modifications. Cells were harvested prior to treatment as the controls, and after 0.5, 2, 4, 8 and 24 hours of

EgKI-1 treatment. Protein extraction and tryptic peptide fragment generation was conducted following a modified FASP protocol for high-throughput sample preparation (27). The data for the ion library generation were obtained using Data Dependent Acquisition (DDA) on each individual samples as previously described (28) while quantitative data from different time points was obtained using SWATH acquisition with the same conditions used in the DDA experiments. A rolling collision energy method was used to fragment all ions in a set of 26 sequential overlapping windows of 25 AMU over a mass range coverage of 350-1,000 $(\mathrm{m} / \mathrm{z})$. Data was acquired and processed using Analyst TF 1.7 software (AB SCIEX).

\section{Protein library generation and Bioinformatic analysis of SWATH} protein quantification

Spectral searches of processed LC-MS/MS data were performed using ProteinPilot v4.5 (AB SCIEX) using the Paragon algorithm (version 4.5.0.0). Background correction was used and biological modifications specified as an ID focus. The detected protein threshold was set as 0.5 and the false-discovery rate (FDR) was calculated using searches against a decoy database comprised of reversed sequences. Searches were conducted against the UniProt human reference proteome set comprising 70953 protein sequences.

For spectral library generation and SWATH XIC peak area extraction PeakView v2.2.0 (AB SCIEX) with the SWATH acquisition MicroApp was used with ion library parameters set to 
6 peptides per protein, 6 transitions per peptides, a peptide confidence threshold of 99\% and FDR threshold to $1 \%$. The XIC time window was set to 6 min and XIC width to $75 \mathrm{ppm}$. All SWATH experiments used iRT calibrants to normalize retention times. To generate the quantitation table files for ions, peptides and proteins, Marker View v1.2.1.1 (AB SCIEX) was used and the relative area under peaks across the different experiments was normalized based on the iRT internal calibrant.

\section{Bioavailability of EgKI-1}

To determine the absorption rate and stability of EgKI-1 in serum, $50 \mu \mathrm{g}$ of the protein in 50 $\mu 1$ of Tris/ $\mathrm{NaCl}$ buffer ( $\mathrm{pH} 7$ ) was injected intraperitoneally into BALB/c mice and blood samples were collected at 5, 30, 120, and $300 \mathrm{~min}$ after injection. The serum was separated and samples analyzed using liquid chromatography-mass spectrometry to monitor the presence of EgKI-1 at the different time points.

\section{In vivo animal model}

MDA-MB-231 cells $\left(1 \times 10^{6}\right)$ were injected into the right inguinal mammary tissue of 6-7 weeks old BALB/c nude mice. When the tumors reached approximately $30 \mathrm{~mm}^{3}$, in 20 days, mice in the control group received buffer only $(150 \mathrm{mM} \mathrm{NaCl}, 20 \mathrm{mM}$ Tris $\mathrm{HCl}, \mathrm{pH} 7)$ and the treatment group were treated with $4 \mathrm{mg} / \mathrm{kg}$ of EgKI-1 per mouse (equivalent to $80 \mu \mathrm{g}$ per $20 \mathrm{~g}$ mouse). EgKI-1 and buffer were injected into the tumors in a total volume of $50 \mu 1$ once a day every other day. All mice were monitored daily and tumor volumes were measured twice a week using digital Vernier caliper and expressed as $\mathrm{mm}^{3}$ according to the formula, a $\times b \times b \times 0.5$, where "a" the length and " $b$ " the measured breadth of the tumor. Mice were also assessed for clinical signs according to an approved clinical score sheet for distress during the course of the experiment (29). Scores for each parameter were summed to give a possible total of 8 . Less than 3 was considered a mild clinical score, between 3-6 a moderate and over 6 was considered a severe clinical score. Experimentation on an individual mouse was terminated when an unacceptable clinical score $(>6)$ was reached, or the cumulative tumor burden of the animal exceeded $1000 \mathrm{~mm}^{3}$. Mice were humanely euthanized by exposure to carbon dioxide at the end of the experiment and tumors were collected.

Paraffin blocks were made with the harvested tumor tissues and sections were stained with mouse monoclonal Ki67 antibodies for immunolabelling screening in the QIMRB histology facility. Ki67 is a nuclear protein that is expressed in proliferating cells and is thus a marker 
for cell proliferation in solid tumors (30). Ki67 stained slides were then scanned at $20 \times$ magnification with an Aperio Scanscope XT slidescanner and digital images were analyzed with ImageScope viewing software. The Aperio nuclear algorithm which is based on the spectral differentiation between brown (positive) and blue counter staining was used for analysis. Total percentage positivity for each slide was then calculated and analyzed using GraphPad Prism.

\section{Animal ethics statement}

This study was performed in strict accordance with protocols approved by the QIMRB Animal Ethics Committee, approval number A1606-617M, which adheres to the Australian code of practice for the care and use of animals for scientific purposes, as well as the Queensland Animal Care and Protection Act 2001; Queensland Animal Care and Protection Regulation 2002. Mice were housed in a specific pathogen free facility with 12 hours light/dark cycle and continual access to food and water.

\section{Statistical analysis}

All data are presented as the means \pm standard mean of error (SEM) of three different experiments. A P-value of $<0.05$ was considered as statistically significant according to the Student's t-test and ANOVA tests. All statistical analysis was performed using GraphPad Prism version 7.

\section{Data availability}

All data generated or analyzed during this study are included in this published article (and its Supplementary Information files).

\section{Results}

\section{Recombinant EgKI-1 expression and purification}

In order to examine the effects of EgKI-1 on cancer cell lines the protein was expressed in yeast. The denaturing SDS-PAGE of the purified EgKI-1 protein revealed a single band around $8 \mathrm{kDa}$ which is consistent with the predicted molecular size of $8.08 \mathrm{kDa}$ (S1 Fig). MALDI-TOF MS analysis with the final purified protein sample showed a $100 \%$ intensity 
peak at $7.506 \mathrm{kDa}$. In-gel digestion identified the protein band as a BPTI/Kunitz inhibitor from E. granulosus with $91 \%$ coverage.

\section{Recombinant EgKI-1 inhibits cell growth in vitro}

To test the effects of EgKI-1 on the proliferation of cancer cell lines, end point and real time monitored cell growth assays were carried out. EgKI-1 treatment inhibited the growth of a range of human cancer cells at different rates when assessed by end point SRB assay (S2 Fig; Table 1). The protein had substantially less effect on the growth of primary neonatal foreskin fibroblast (NFF) cells, and had $\sim 9-40$ fold high $\mathrm{IC}_{50}$ value compared with the $\mathrm{IC}_{50}$ values of tested human cancer cell lines.

Real time monitoring using the IncuCyte showed that cells treated with $1 \mu \mathrm{M}$ EgKI-1 eventually lost their typical shape, lost their ability to proliferate with time (Fig 1a), and exhibited dose-dependent growth inhibition compared with the control cells (Fig 1b). Furthermore, the IncuCyte analysis showed that cells continued to grow in the presence of EgKI-1, at a concentration of around $0.5 \mu \mathrm{M}$, before starting to die after $\sim 60$ hours making it a more sensitive assay than end point staining.

\section{EgKI-1 treatment inhibits cell migration}

We examined the effect of EgKI-1 treatment on cell migration on a panel of representative cell lines. The real time scratch wound assay analysis using the IncuCyte system revealed that $0.5 \mu \mathrm{M}$ EgKI-1 inhibited the migration of the CJM (melanoma), MDA-MB-231 (breast cancer) and HeLa (cervical cancer) cells in vitro (Fig 2a). The gap in the scratch wound in control wells decreased eventually with time. The wound width was significantly larger in EgKI-1-treated wells compared with controls after 2 days indicating the inhibition of cell migration by EgKI-1. According to the analysis, the closure of the scratch in EgKI-1-treated cells were $12 \%$ in CJM, $18 \%$ in MDA-MB-231 and 16\% in HeLa compared with $60 \%$, 58\% and $100 \%$ in the controls respectively (Fig 2b). This data indicates that EgKI-1 inhibits cancer cell migration.

\section{Immunocytochemistry demonstrates EgKI-1 internalization}

The presence of green fluorescence in the cells indicated that cancer cells internalized the EgKI-1 protein after treatment. Furthermore, vacuoles started to appear in the cytoplasm, 
which can lead to cell membrane collapse and eventually necrosis. There were significant decreases in the intensity of tubulin and total cell area indicating the degradation of the cytoskeleton following treatment with the EgKI-1 protein (Fig 3).

\section{EgKI-1 disrupts cell cycle profile}

Cell cycle profiles of MDA-MB-231 and HeLa cells were assessed after EgKI-1 treatment. Even though significant non-adherent cells were observed only attached cells were harvested and assessed. EgKI-1 treatment caused a significant increase in S phase in both MDA-MB231 and HeLa cell lines 2 days post-treatment. MDA-MB-231 cells treated with EgKI-1 (2 $\mu \mathrm{M})$ significantly decreased the $\mathrm{G} 2 / \mathrm{M}$ phase $(\sim 60 \%)$ by arresting the proliferative $\mathrm{S}$ phase of the cell cycle at 2 days post-treatment compared with the control cells (Fig 4). In HeLa cells, EgKI-1 perturbed cell cycle progression by significantly decreasing the non-proliferative $\mathrm{G} 0 / \mathrm{G} 1$ fraction $(\sim 30 \%)$ and increasing the $\mathrm{S}(\sim 30 \%)$ and $\mathrm{G} 2 / \mathrm{M}(\sim 80 \%)$ fractions 2 days posttreatment (Fig 4). No significant difference was observed 1 day post-treatment (S3 Fig).

\section{EgKI-1 can induce apoptosis}

As we observed a significant proportion of non-adherent cells following EgKI-1 treatment, treated cells were assessed for apoptosis. EgKI-1 induced apoptosis in MDA-MB-231 breast cancer cells 24 hours post-treatment in a dose-dependent manner. Cells treated with 0.2 $\mu \mathrm{g} /$ well of EgKI-1 $(2 \mu \mathrm{M})$ had a significantly higher percentage of cells $(19.9 \%)$ in early apoptosis compared with control cells whereas cells treated with $0.8 \mu \mathrm{g} / \mathrm{well}$ EgKI-1 ( $0.5 \mu \mathrm{M}$ concentration) had a higher percentage of cells (78.6\%) in late apoptosis (Fig 5).

\section{Proteomics analysis following EgKI-1 treatment}

SWATH-MS analysis identified a total of 1770 proteins (shown in the heat map; S4 Fig) in cancer cells. EgKI-1 treatment mainly up-regulated Bcl-2-like protein 13 (Q9BXK5) and inhibitor of nuclear factor kappa-B kinase subunit beta (O14920) expression with time. Tetraspanin (H7BXY6), nucleoside diphosphate kinase A (P15531) and double-strand-break repair protein (O60216) expression were down-regulated with time (S5 Fig).

\section{Bioavailability of EgKI-1}


When injected intraperitoneally into mice, the EgKI-1 protein was absorbed into the blood in 5 minutes and the highest serum availability occurred within 30 minutes. Most of the EgKI-1 protein had been cleared from the blood by 5 hours (S6 Fig). No adverse or toxic reactions resulting from the administration of EgKI-1 to the mice occurred.

\section{EgKI-1 reduces tumor growth in an in vivo breast cancer model}

We assessed the impact of EgKI-1 intra-lesional treatment on the growth of MDA-MB-231 tumors in vivo. The tumors were allowed to reach approximately $30 \mathrm{~mm}^{3}$ before treatment with $4 \mathrm{mg} / \mathrm{kg}$ of EgKI-1, or equivalent vehicle, once per day every other day. The growth of MDA-MB-231 breast tumors was significantly reduced by $57.7 \%$ in EgKI-1-treated mice compared with control mice (Fig 6). Furthermore, expression of Ki67 protein, which is a proliferation marker, was significantly reduced in the EgKI-1-treated tumor tissues indicating $\sim 80 \%$ reduction of tumor proliferation (Fig 6 ) as suggested by the in vitro data.

\section{Discussion}

This study describes the broad-spectrum anti-cancer activities of EgKI-1 which is the first anti-cancer molecule identified from the cestode E. granulosus. Although we hypothesized based on our previous studies (16) that the potent neutrophil elastase activity of EgKI-1 would result in cancer cell regression, the in vitro culture medium we employed did not contain neutrophils or neutrophil elastase (S2 Fig). Therefore there must be additional mechanisms whereby EgKI-1 adversely affects cancer cell growth. Inhibition of the growth of the different cancer cell lines with an $\mathrm{IC}_{50}$ range of $1.1-5.1 \mu \mathrm{M}$, with only minimal reduction in cell growth of primary neonatal fibroblasts, suggests that EgKI-1 potentially interacts with cancer-specific proteins only. EgKI-1 treatment specifically inhibited the growth of invasive, rapid proliferative cell lines like MCF-7, FaDu and HeLa with lower $\mathrm{IC}_{50}$ values compared with other tested cell lines $(31,32)$. Cancer cell migration can lead to tumor metastasis and invasion (33). Therefore the observed inhibition of cancer cell migration by EgKI-1 is also an important aspect to consider for cancer therapeutic development.

Further, the apparent cell growth inhibition by EgKI-1 was due to an increase in apoptosis, potentially by disrupting cell cycle progression. Both HeLa and MDA-MB-231 exhibited increased numbers of cells in $\mathrm{S}$ phase of the cell cycle following treatment. While MDA-MB231 showed a reduction of cell numbers in G2/M transition, HeLa had increased G2/M 
numbers following treatment. Abnormal, deregulated cancer cells undergo unrestricted division which is different from other cancers and, as an "immortal" cell line, HeLa cells divide differently (34). This can possibly be a reason for the changes in the cell cycle of HeLa and MDA-MB-231 cell lines treated with EgKI-1. Identifying new cancer-specific molecules that can target mitosis can optimize combinatorial treatments in the future (35). The induction of apoptosis by EgKI-1 at the same concentration that led to 50\% inhibition in cell growth/proliferation shows the effectiveness of the protein in cancer cell growth inhibition. Apoptosis-targeted cancer therapy has undoubtedly been an indispensable approach in treating cancer, in order to make damaged cells commit suicide (36). The immunocytochemistry results presented here indicated that EgKI-1 was internalized in cancer cells. The immediate effects on the plasma membrane evident after EgKI-1 treatment might therefore support the sequence of events involving EgKI-1 in the mechanism of action leading to the induction of apoptosis.

SWATH-MS analysis supports the relative quantification of large fractions of a proteome in a single sample (37). EgKI-1 treatment up-regulated BcL-2 like protein 13 (UniProt IDQ9BXK5) which promotes the activation of caspase-3 and apoptosis (38). EgKI-1 treatment further down-regulated the expression of tetraspanin (UniProt ID-H7BXY6) and BRCA1associated ATM activator-1 (BRAT1) (UniProt ID-Q6PJG6) proteins. Tetraspanins can promote multiple cancer stages by playing key roles in tumor initiation and metastasis (39). BRAT1 plays broad roles in DNA repair and cell cycle regulation involved in controlling cell growth (40). Therefore, down-regulation of tetraspanin and BRAT1, caused by EgKI-1, can contribute to cancer cell growth inhibition but additional study is required to identify the precise molecules and mechanisms of action of EgKI-1 in cancer cell growth inhibition.

Metastatic breast cancer is the leading cause of cancer death in women worldwide (41). Therefore, the MDA-MB-231 cell line was selected for further investigation and in in vivo animal model studies. In this study we used intra-tumor delivery of EgKI-1 as direct injection into a tumor lesion has the advantage that much higher drug concentrations can be applied at the tumor site with the result that intra-lesional anti-tumor therapeutics are considered as more effective than other routes (42). After 26 days of EgKI-1 treatment there was $\sim 60 \%$ reduction of tumor growth in treated mice compared with controls. As Ki67 expression is strongly associated with tumor cell proliferation (30), the significantly decreased numbers of Ki67-positive cells in tumor tissues treated with EgKI-1 reflect its inhibitory effect on cell proliferation in vivo. 
A therapeutic with a molecular size less than $10 \mathrm{kDa}$ allows rapid extravasation from blood vessels and rapid transport to tumor targets resulting in maximal tumor uptake (43). However, such molecules have shorter serum half-lives than longer peptides as they are quickly cleared by renal filtration (44). PEGylation, the technique of covalently attaching polyethylene glycol (PEG) to a molecule, which may be a low molecular size protein, enzyme or nanoparticle, has proven to be one of the best methods for the passive targeting of anti-cancer therapeutics $(45,46)$. Accordingly, this technology will be applied in the future to improve the serum half-life of EgKI-1 if needed.

Another future consideration will be to monitor the up or down regulation of genes involved in cancer progression and metastasis following EgKI-1 treatment. Further, EgKI-1 treatment can inhibit the cellular activity of different proteases, including trypsin, plasma kallikrein and cathepsins, and matrix metalloproteases as analyzed with P237, P139 and P126 fluorescence substrates (unpublished data). We have also shown that EgKI-1 can induce some antiinflammatory cytokines (unpublished data), which is important as chronic inflammation increases the risk of cancer and a reduction in inflammation helps in cancer therapy (47). Therefore immunomodulation effects induced by EgKI-1 may also play a role in cancer growth inhibition in vivo, an area that needs to be explored in the future using immunocompetent mouse models of cancer.

\section{Conclusion}

The selective killing of malignant cells without affecting normal cells is the ultimate goal of cancer therapy. As a low molecular weight $(<10 \mathrm{kDa})$ protein, EgKI-1 has potential to penetrate tumor tissues for effective interaction and killing of malignant cells. EgKI-1 treatment significantly reduced the rate of breast cancer growth in vivo and represents a promising molecule for development as a future anti-cancer therapeutic.

\section{References}

1. Kintzing JR, Filsinger Interrante MV, Cochran JR. Emerging Strategies for Developing Next-Generation Protein Therapeutics for Cancer Treatment. Trends Pharmacol Sci. 2016;37(12):993-1008.

2. Craik DJ, Fairlie DP, Liras S, Price D. The future of peptide-based drugs. Chem Biol Drug Des. 2013;81(1):136-47.

3. Srikanth S, Chen Z. Plant Protease Inhibitors in Therapeutics-Focus on Cancer Therapy. Front Pharmacol. 2016;7:470. 
4. Hewitson JP, Grainger JR, Maizels RM. Helminth immunoregulation: the role of parasite secreted proteins in modulating host immunity. Mol Biochem Parasitol. 2009;167(1):1-11.

5. van Tong H, Brindley PJ, Meyer CG, Velavan TP. Parasite Infection, Carcinogenesis and Human Malignancy. EBioMedicine. 2017;15:12-23.

460 Trypanosoma cruzi surface molecule gp82 induces apoptotic cell death in melanoma cells. Melanoma Res. 2008;18(3):172-83.

462 7. Shirzad H, Khorami S, Soozangar N, Yousefi M, Darani HY. Toxoplasma gondii but not Leishmania major or Trichomonas vaginalis decreases cell proliferation and increases cell death on fibrosarcoma cancer cells in culture medium. 2012.

8. Yousofi Darani H, Soozangar N, Khorami S, Taji F, Yousofi M, Shirzad H. Hydatid Cyst Protoscolices Induce Cell Death in WEHI-164 Fibrosarcoma Cells and Inhibit the Proliferation of Baby Hamster Kidney Fibroblasts In Vitro. J Parasitol Res. 2012;2012:304183.

9. Uribe-Querol E, Rosales C. Neutrophils in Cancer: Two Sides of the Same Coin. J Immunol Res. 2015;2015:983698. ratio is associated with breast cancer prognosis: an updated systematic review and metaanalysis. OncoTargets and therapy. 2016;9:5567.

474 11. Coffelt SB, Wellenstein MD, de Visser KE. Neutrophils in cancer: neutral no more. Nature Reviews Cancer. 2016;16(7):431-46.

476 12. Young RE, Voisin MB, Wang S, Dangerfield J, Nourshargh S. Role of neutrophil elastase in LTB4-induced neutrophil transmigration in vivo assessed with a specific inhibitor and neutrophil elastase deficient mice. Br J Pharmacol. 2007;151(5):628-37. 13. Gregory AD, Houghton AM. Tumor-associated neutrophils: new targets for cancer therapy. Cancer Res. 2011;71(7):2411-6.

481 14. Craig P, Rogan M, Campos-Ponce M. Echinococcosis: disease, detection and transmission. Parasitology. 2003;127(S1):S5-S20.

483 15. McManus DP, Zhang W, Li J, Bartley PB. Echinococcosis. The Lancet. $484 \quad 2003 ; 362(9392): 1295-304$. Characterization of Two Potent Kunitz Type Protease Inhibitors from Echinococcus granulosus. PLoS Negl Trop Dis. 2015;9(12):e0004268.

488 17. RANASINGHE S, MCMANUS D, Zhang W, Fischer K. Treatment of inflammation and/or cancer. Google Patents; 2016.

490 18. Bergstrom FC, Reynolds S, Johnstone M, Pike RN, Buckle AM, Kemp DJ, et al. 491 Scabies mite inactivated serine protease paralogs inhibit the human complement system. J Immunol. 2009;182(12):7809-17.

19. Kruger NJ. The Bradford method for protein quantitation. Methods Mol Biol. 1994;32:9-15. spectrometric characterization of proteins and proteomes. Nat Protoc. 2006;1(6):2856-60. 21. Drexler HG, Uphoff CC. Mycoplasma contamination of cell cultures: Incidence, sources, effects, detection, elimination, prevention. Cytotechnology. 2002;39(2):75-90.

22. Skehan P, Storeng R, Scudiero D, Monks A, McMahon J, Vistica D, et al. New colorimetric cytotoxicity assay for anticancer-drug screening. J Natl Cancer Inst. 1990;82(13):1107-12.

23. Liang CC, Park AY, Guan JL. In vitro scratch assay: a convenient and inexpensive method for analysis of cell migration in vitro. Nat Protoc. 2007;2(2):329-33. 
24. Schindelin J, Arganda-Carreras I, Frise E, Kaynig V, Longair M, Pietzsch T, et al. Fiji: an open-source platform for biological-image analysis. Nature methods. 2012;9(7):67682.

25. Riccardi C, Nicoletti I. Analysis of apoptosis by propidium iodide staining and flow cytometry. Nature protocols. 2006;1(3):1458.

26. Gillet LC, Navarro P, Tate S, Rost H, Selevsek N, Reiter L, et al. Targeted data extraction of the MS/MS spectra generated by data-independent acquisition: a new concept for consistent and accurate proteome analysis. Mol Cell Proteomics. 2012;11(6):O111 016717.

27. Potriquet J, Laohaviroj M, Bethony JM, Mulvenna J. A modified FASP protocol for high-throughput preparation of protein samples for mass spectrometry. PLoS One. 2017;12(7):e0175967.

28. Ponce D, Brinkman DL, Potriquet J, Mulvenna J. Tentacle Transcriptome and Venom Proteome of the Pacific Sea Nettle, Chrysaora fuscescens (Cnidaria: Scyphozoa). Toxins (Basel). 2016;8(4):102.

29. Boyle GM, D'Souza MM, Pierce CJ, Adams RA, Cantor AS, Johns JP, et al. Intralesional injection of the novel PKC activator EBC-46 rapidly ablates tumors in mouse models. PLoS One. 2014;9(10):e108887.

30. Li LT, Jiang G, Chen Q, Zheng JN. Ki67 is a promising molecular target in the diagnosis of cancer. Molecular medicine reports. 2015;11(3):1566-72.

31. Comsa S, Cimpean AM, Raica M. The Story of MCF-7 Breast Cancer Cell Line: 40 years of Experience in Research. Anticancer Res. 2015;35(6):3147-54.

32. Qi W, Zhao C, Zhao L, Liu N, Li X, Yu W, et al. Sorting and identification of side population cells in the human cervical cancer cell line HeLa. Cancer Cell Int. 2014;14(1):3.

33. Paul CD, Mistriotis P, Konstantopoulos K. Cancer cell motility: lessons from migration in confined spaces. Nat Rev Cancer. 2017;17(2):131-40.

34. Ivankovic M, Cukusic A, Gotic I, Skrobot N, Matijasic M, Polancec D, et al. Telomerase activity in HeLa cervical carcinoma cell line proliferation. Biogerontology. 2007;8(2):163-72.

35. Chan K, Koh CG, Li H. Mitosis-targeted anti-cancer therapies: where they stand. Cell death \& disease. 2012;3(10):e411.

36. Baig S, Seevasant I, Mohamad J, Mukheem A, Huri H, Kamarul T. Potential of apoptotic pathway-targeted cancer therapeutic research: Where do we stand? Cell death \& disease. 2016;7(1):e2058.

37. Schubert OT, Gillet LC, Collins BC, Navarro P, Rosenberger G, Wolski WE, et al. Building high-quality assay libraries for targeted analysis of SWATH MS data. Nat Protoc. 2015;10(3):426-41.

38. Pellegrini M, Strasser A. Caspases, Bcl-2 Family Proteins and Other Components of the Death Machinery: Their Role in the Regulation of the Immune Response. 2013.

39. Hemler ME. Tetraspanin proteins promote multiple cancer stages. Nature reviews Cancer. 2014;14(1):49.

40. So EY, Ouchi T. The potential role of BRCA1-associated ATM activator-1 (BRAT1) in regulation of mTOR. Journal of cancer biology \& research. 2013;1(1).

41. Wahba HA, El-Hadaad HA. Current approaches in treatment of triple-negative breast cancer. Cancer Biol Med. 2015;12(2):106-16.

42. Toomey P, Kodumudi K, Weber A, Kuhn L, Moore E, Sarnaik AA, et al. Intralesional injection of rose bengal induces a systemic tumor-specific immune response in murine models of melanoma and breast cancer. PLoS One. 2013;8(7):e68561.

43. Schmidt MM, Wittrup KD. A modeling analysis of the effects of molecular size and binding affinity on tumor targeting. Mol Cancer Ther. 2009;8(10):2861-71. 
44. Wittrup KD, Thurber GM, Schmidt MM, Rhoden JJ. Practical theoretic guidance for the design of tumor-targeting agents. Methods Enzymol. 2012;503:255-68.

45. Mishra P, Nayak B, Dey R. PEGylation in anti-cancer therapy: An overview. asian journal of pharmaceutical sciences. 2016;11(3):337-48.

46. Mattheolabakis G, Wong CC, Sun Y, Amella CA, Richards R, Constantinides PP, et al. Pegylation improves the pharmacokinetics and bioavailability of small-molecule drugs hydrolyzable by esterases: a study of phospho-Ibuprofen. J Pharmacol Exp Ther. 2014;351(1):61-6.

47. Rayburn ER, Ezell SJ, Zhang R. Anti-Inflammatory Agents for Cancer Therapy. Mol Cell Pharmacol. 2009;1(1):29-43.

\section{Author Contributions Statement}

SLR, GMB, JPM and DPM planned the study. SLR, KF and JP performed the experiments, analyzed the data and prepared figures. GMB and DPM provided guidance to the study and corrected the manuscript. All authors reviewed the manuscript.

\section{Competing Financial Interests}

The authors declare that they have no competing interests.

\section{Financial Support}

This project was funded by program (APP1037304 to DPM) from the Australian National Health and Medical Research Council (NHMRC). DPM is a NHMRC Senior Principal Research Fellow and Senior Scientist at QIMR Berghofer.

Fig 1: Growth of cancer cells inhibited by EgKI-1. (a) CJM, MDA-MB-231 and HeLa cells lost shape and started dying when treated with $1 \mu \mathrm{M} \mathrm{EgKI-1}(0.8 \mu \mathrm{g}$ in $100 \mu \mathrm{l} /$ well) over time. (b) Dose-dependent growth inhibition curves with varying concentrations of EgKI1.

Fig 2: EgKI-1 inhibits cancer cell migration in vitro. In the control wells the gap closed with time but in the treated wells the gap closure was significantly reduced compared with the controls. $\mathrm{P}<0.01 * *$ and $\mathrm{p}<0.0001 * * * *$ 
Fig 3: Immunocytochemistry. (a) Control cells; (b) EgKI-1 treated MDA-MB-231 breast cancer cells 24 hours post-treatment. White arrow shows the green in treated cells indicating the EgKI-1 protein; White dotted arrow indicates the vacuole in the cell; Red indicates tubulin in cytoplasm; Blue, DAPI nuclear stain; (c) Cell area reduction ( $<<0.001 * * *)$ and (d) Red fluorescence intensity of tubulin $(\mathrm{p}<0.0001 * * * *)$ after analysis by Student's t-test.

Fig 4: EgKI-1 induces cell cycle arrest. MDA-MB-231 and HeLa cells were treated with 1 $\mu \mathrm{M}$ and $2 \mu \mathrm{M}$ EgKI-1 for 48 hours and the cell cycle distribution was determined by flow cytometry. Results represent the mean \pm SEM from 3 independent experiments. $p<0.05 *$, $\mathrm{p}<0.01 * *, \mathrm{p}<0.001 * * *$ and $\mathrm{p}<0.0001 * * * *$ by 2 way ANOVA test.

\section{Fig 5: Induction of apoptosis in MDA-MB-231 breast cancer cells following EgKI-1} treatment. (a) Control, untreated cells were alive, being Annexin $\mathrm{V}^{-} / \mathrm{PI}^{-}$; (b) $20 \%$ of cells treated with the lower EgKI-1 concentration $(0.25 \mu \mathrm{M})$ were in early apoptosis (Annexin $\left.\mathrm{V}^{+} / \mathrm{PI}\right)$; (c) Cells treated with the higher EgKI-1 $(1 \mu \mathrm{M})$ concentration exhibited the highest percentage of late apoptotic/ early necrotic cells $\left(\right.$ Annexin $\left.\mathrm{V}^{+} / \mathrm{PI}^{+}\right)$.

Fig 6: Anti-tumor effects of EgKI-1 treatment in vivo. (a) Tumor growth curve of MDAMB-231 tumor bearing mice receiving EgKI-1 treatment (4 mg/kg) compared with control mice $\left(\mathrm{P}<0.05^{*}\right.$ by Student's t-test). (b) Tumor size of EgKI-1 treated $(\mathrm{n}=9)$ and control mice $(\mathrm{n}=6)$ surviving at the end of the experiment $(\mathrm{P}<0.05 *$ by Student's $\mathrm{t}$-test $)$ showing $\sim 60 \%$ reduction of tumor growth in treated mice. (c) Representative bright field images for the immunohistochemical staining of Ki67 proliferation marker (brown) in control and treated tumors (scale bar indicates $200 \mu \mathrm{m}$ ). (d) Quantification of Ki67-positive cells $\left(\mathrm{P}<0.05^{*}\right.$ by Student's t-test).

Table 1: IC $_{50}$ values for different human cancer cell lines tested with EgKI-1

\begin{tabular}{ccccccccc}
\hline \multicolumn{7}{c}{ IC $_{\mathbf{5 0}}$ values $(\boldsymbol{\mu M})$ with 95\% CI } \\
\hline Cell line & NFF & MCF-7 & MDA-MB-231 & CJM & SCC15 & FaDu & HeLa & T47D \\
\hline & 47.134 & 2.075 & 5.162 & 4.660 & 4.025 & 2.674 & 1.157 & 3.012 \\
& 18.2 to & 1.88 to & 4.16 to 6.10 & 3.12 to & 3.69 to & 2.17 to & 0.9 to & 2.64 to \\
59.4 & 2.27 & & 8.33 & 4.41 & 3.28 & 1.49 & 3.36 \\
\hline
\end{tabular}

CI - Confidence Interval 


\section{Supporting Information}

617 S1 Fig: Purified recombinant EgKI-1 on an SDS-PAGE gel. 1, $3 \mu$ g EgKI-1; L, molecular 618 size markers; 2, $6 \mu \mathrm{g}$ EgKI-1.

S2 Fig: Viability of different cancer cell lines in the presence of EgKI-1. Viable cell 621 percentages of primary neonatal foreskin fibroblast cells (NFF), breast cancer cell lines 622 (MCF-7, MDA-MB-231, T47D), melanoma (CJM), squamous cell carcinomas (SCC15, $623 \mathrm{FaDu})$ and cervical adenocarcinoma (HeLa) cell lines with different EgKI-1 concentrations in 624 vitro.

S3 Fig: EgKI-1 effect on cell cycle arrest. MDA-MB-231 and HeLa cells were treated with $1 \mu \mathrm{M}$ and $2 \mu \mathrm{M}$ EgKI-1 for 24 hours and the cell cycle distribution was determined by flow cytometry. No significant difference was observed among the different cell cycle phases.

S4 Fig: Differentially expressed proteins quantified by SWATH-MS analysis. Heat map analysis of 1770 proteins among three biological replicates of the control and EgKI-1 treated samples after $30 \mathrm{~min}, 2$ hours, 4 hours and 24 hours. The fold change value of the MS signal intensity is shown.

S6 Fig: Serum stability of EgKI-1. After intraperitoneal injection into mice, EgKI-1 was absorbed into the blood within 5 minutes and had been cleared in 5 hours. 


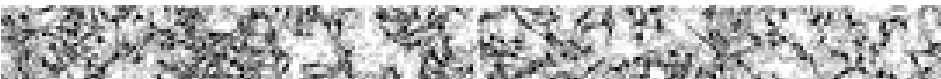
gengentrol

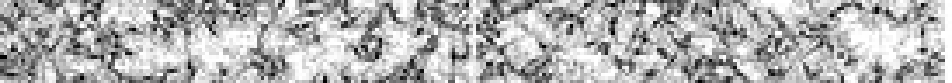

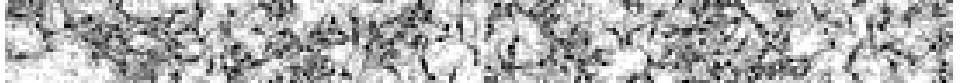

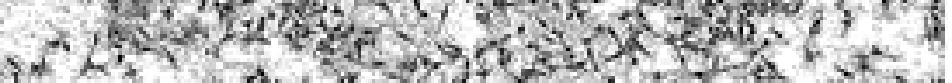

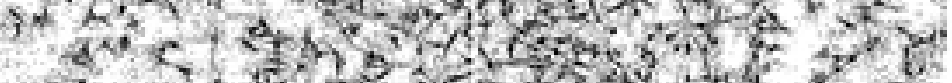

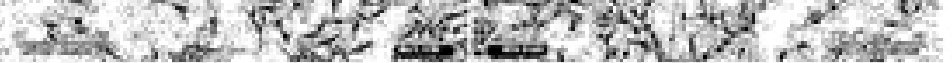

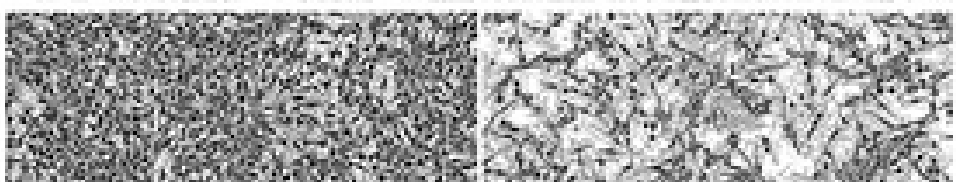

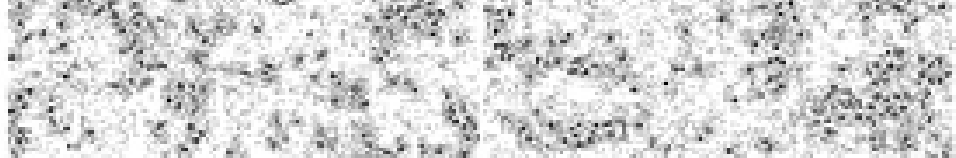

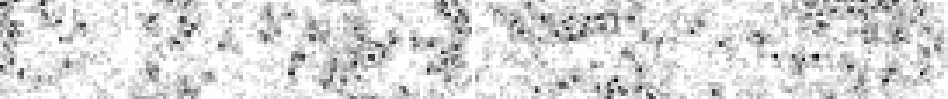

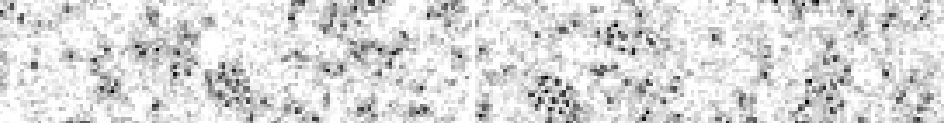

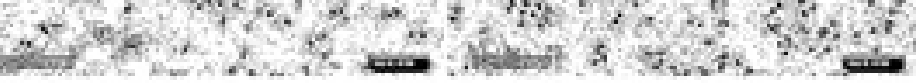

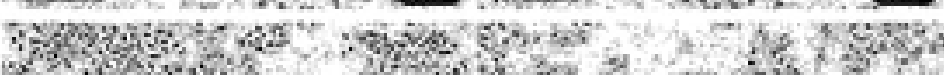

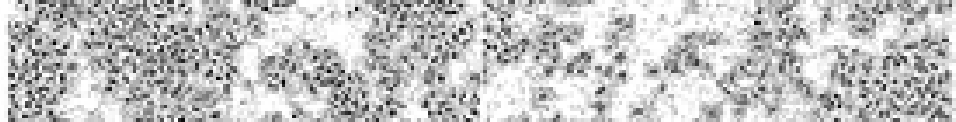

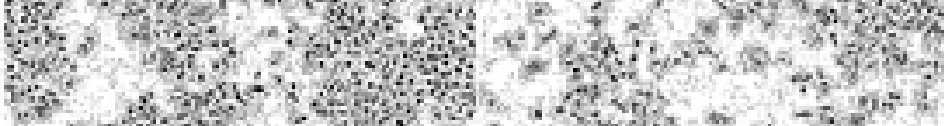

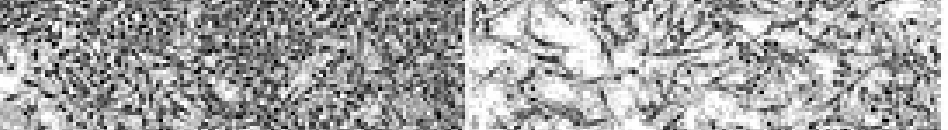

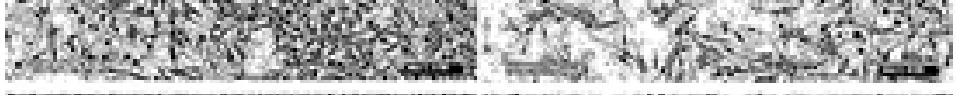

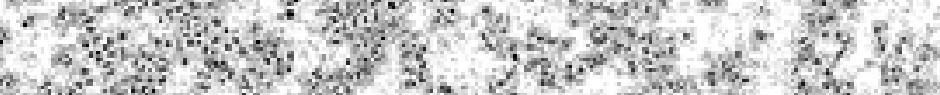

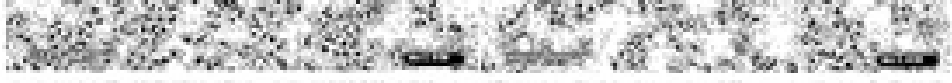
39.

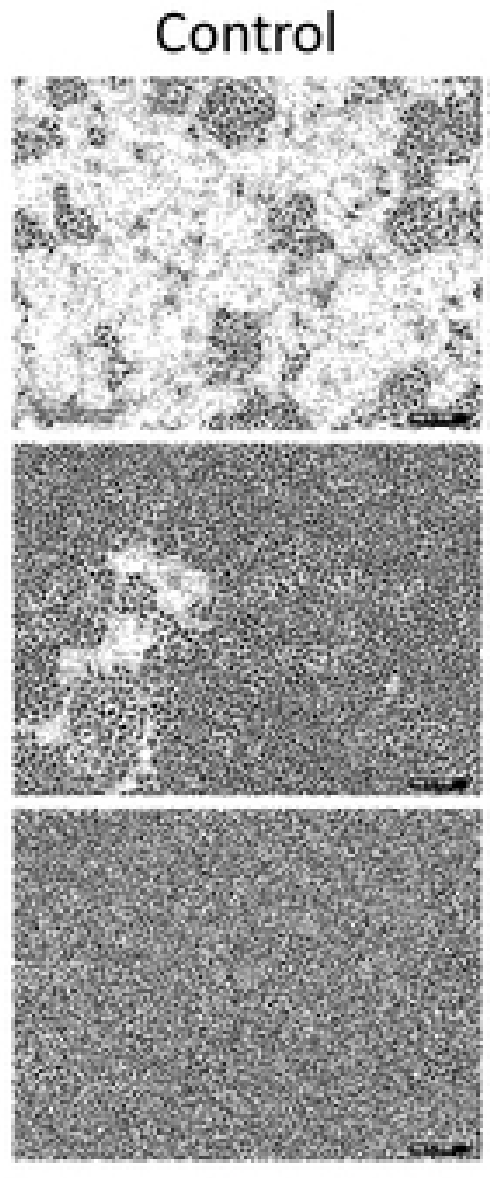
EgKI-1 treated

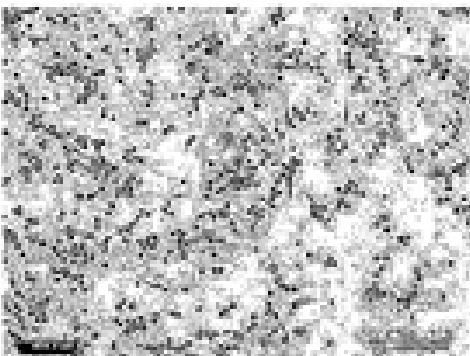

b

CJM

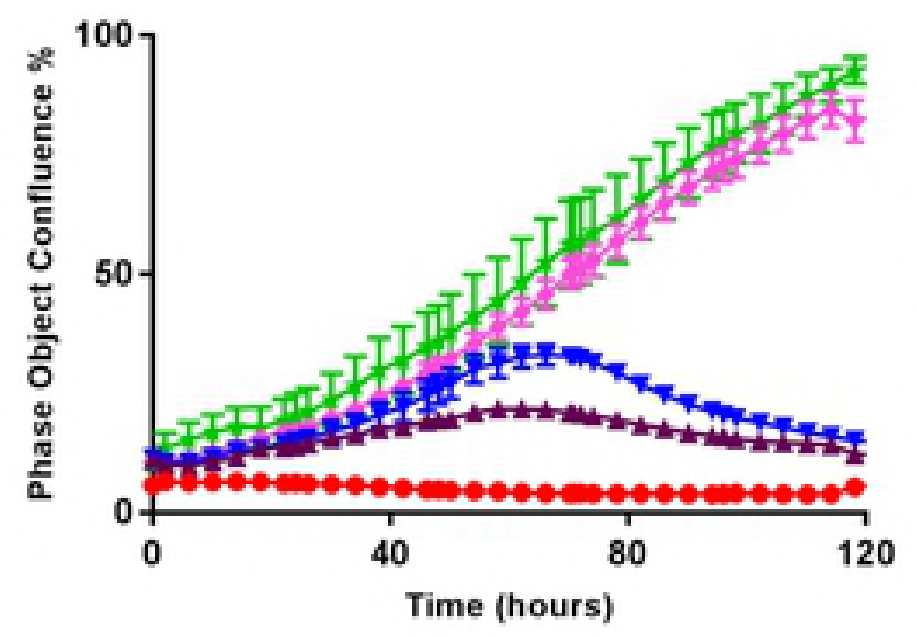

MDA-MB-231

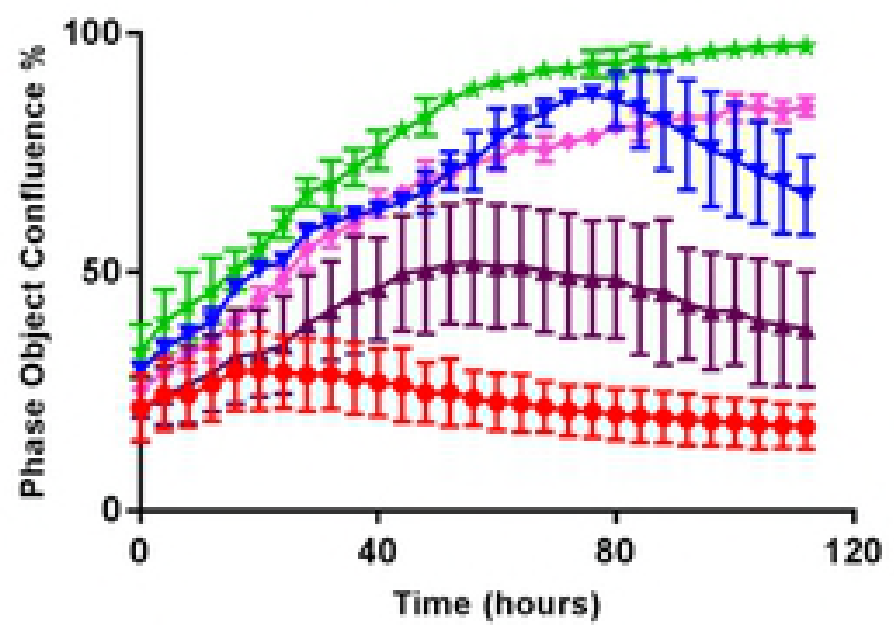

HeLa

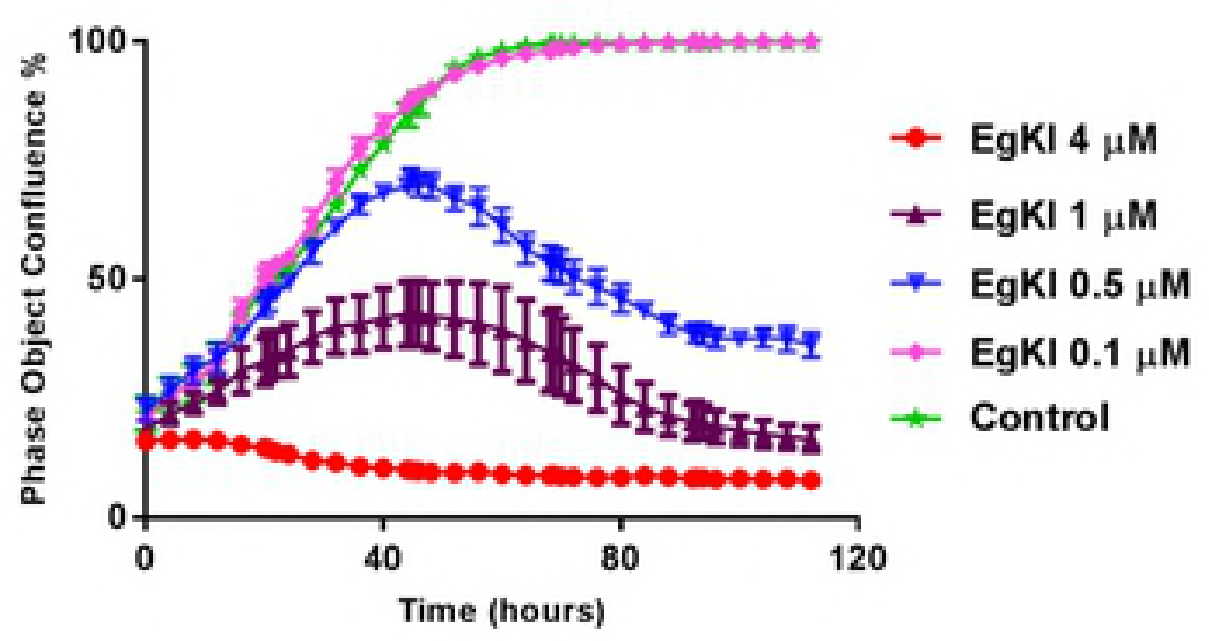




\section{HeLa}

a

\section{Control \\ EgKI-1 treated}

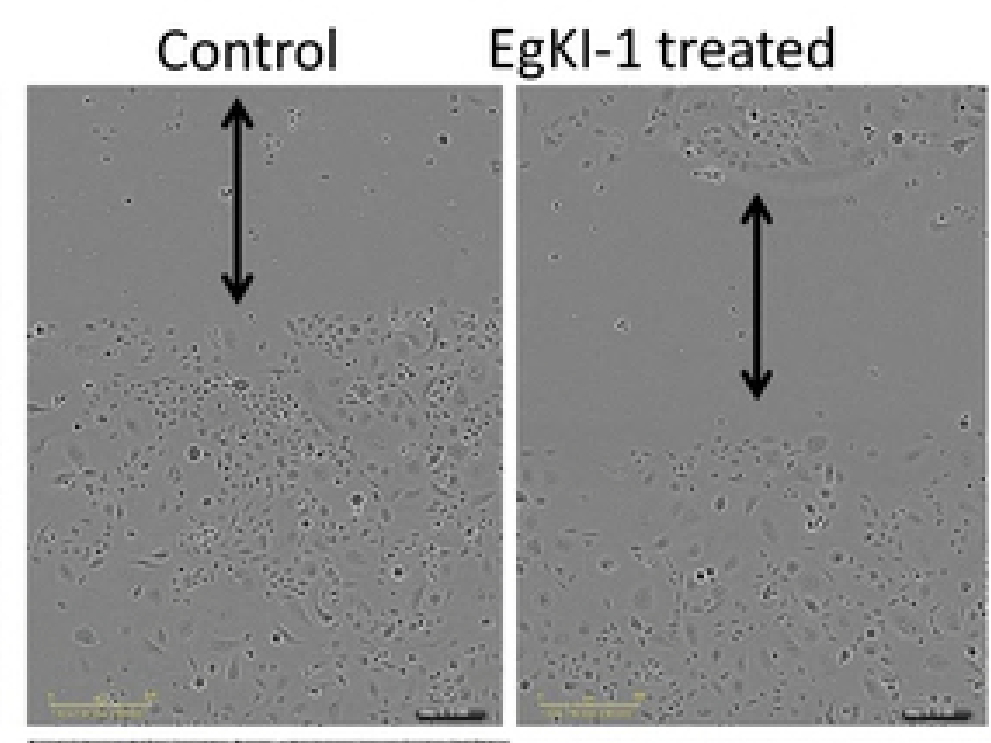

Control

EgKI-1 treated

Od
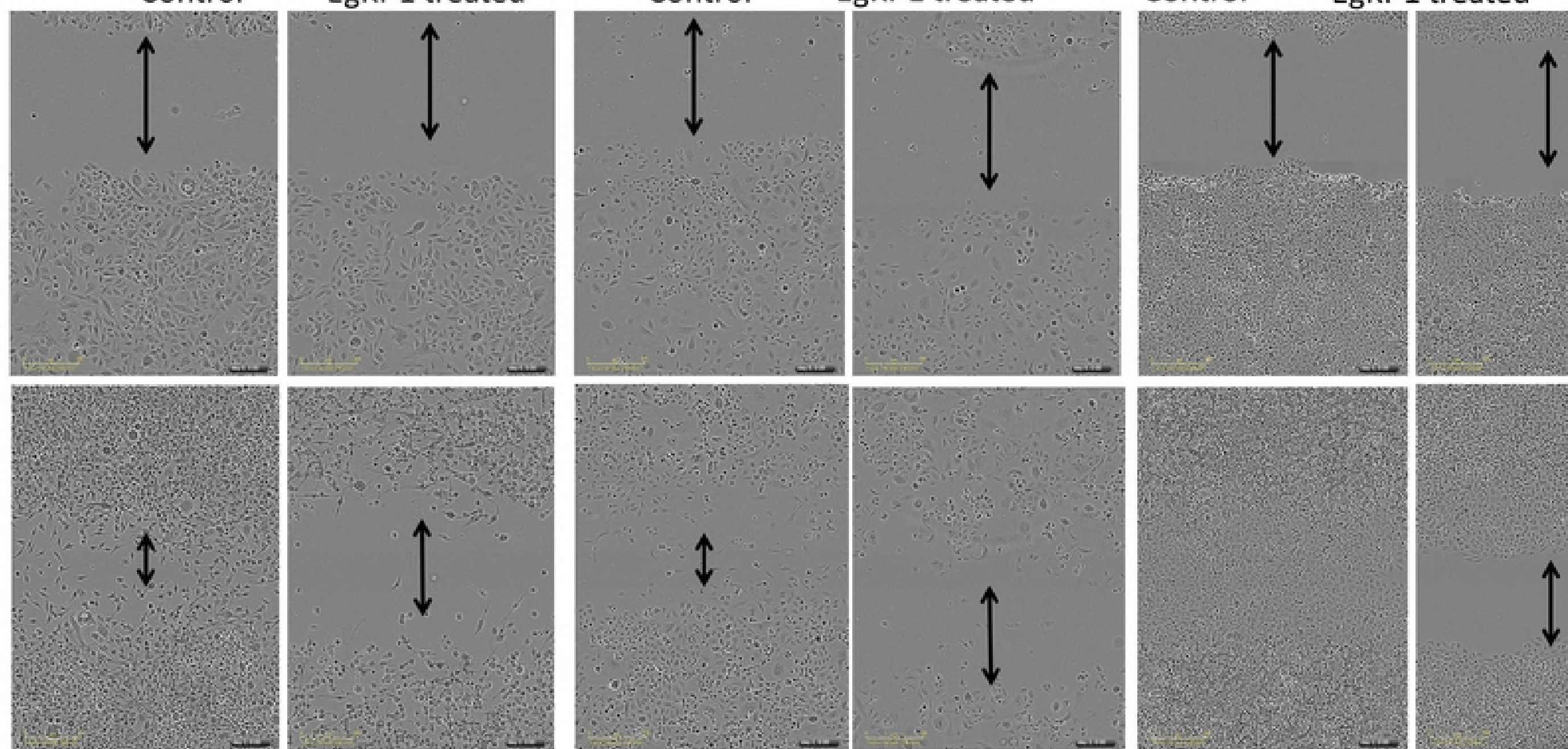

$2 \mathrm{~d}$
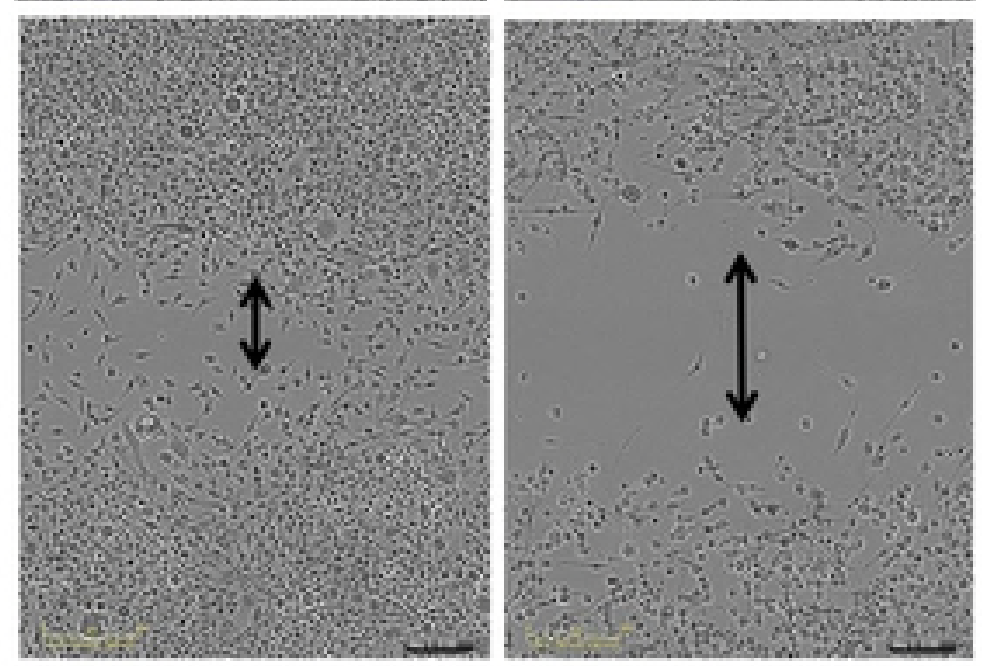

b

CJM

MDA-MB-231

HeLa
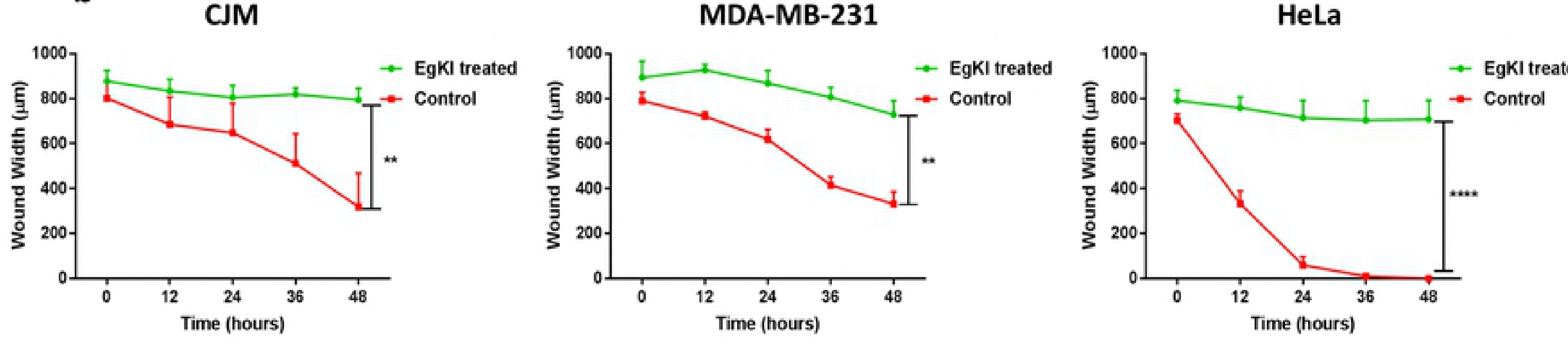
MDA-MB-231 Day 2 post-treatment

HeLa Day 2 post-treatment
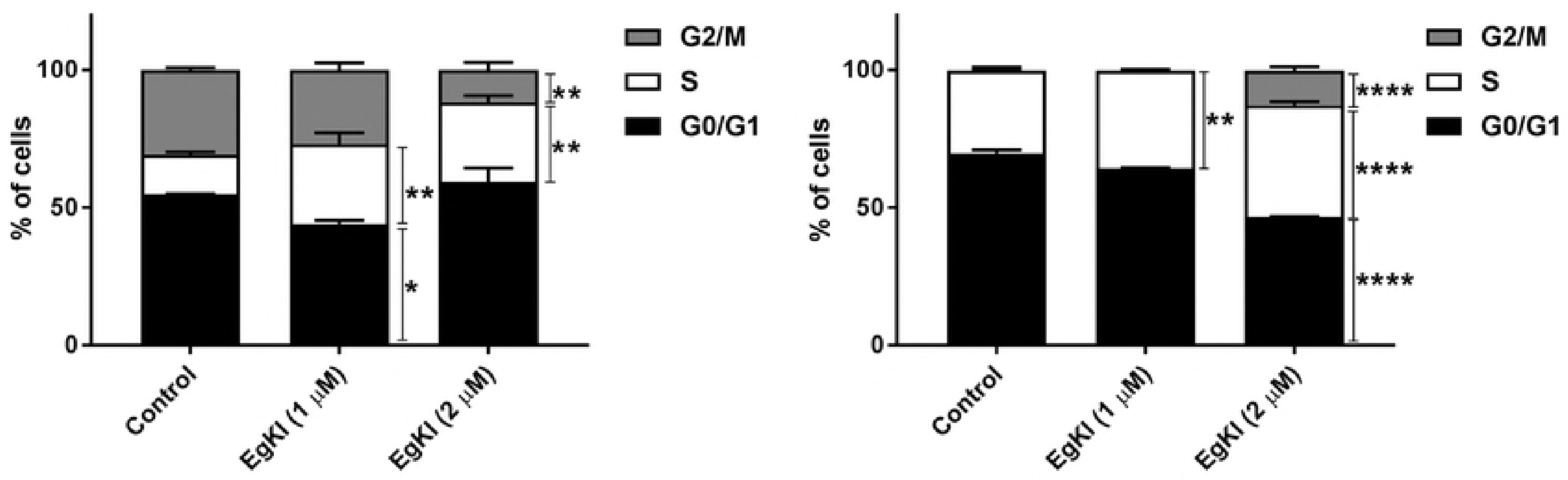

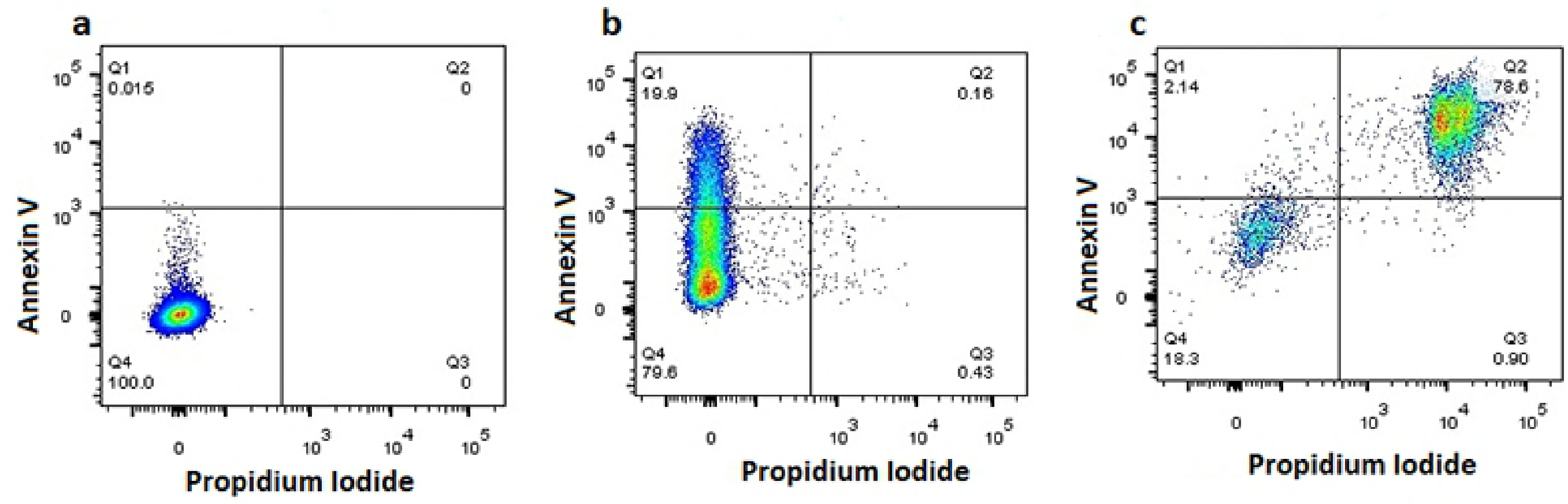

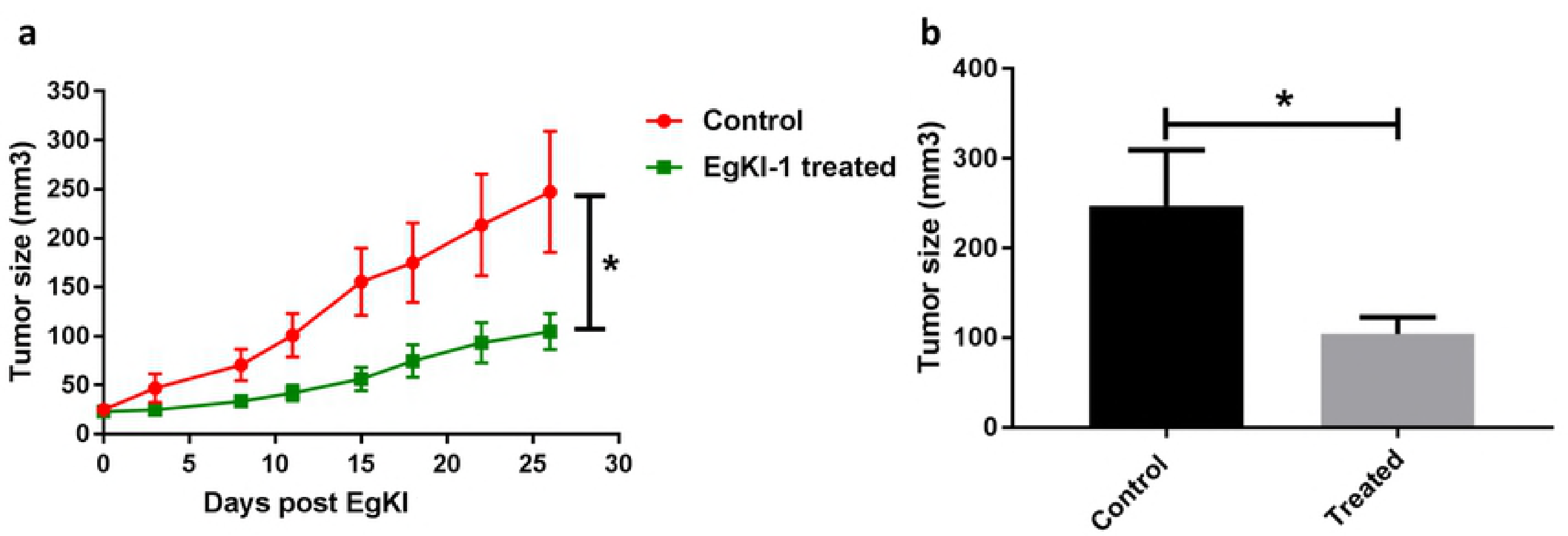

\section{C}

d

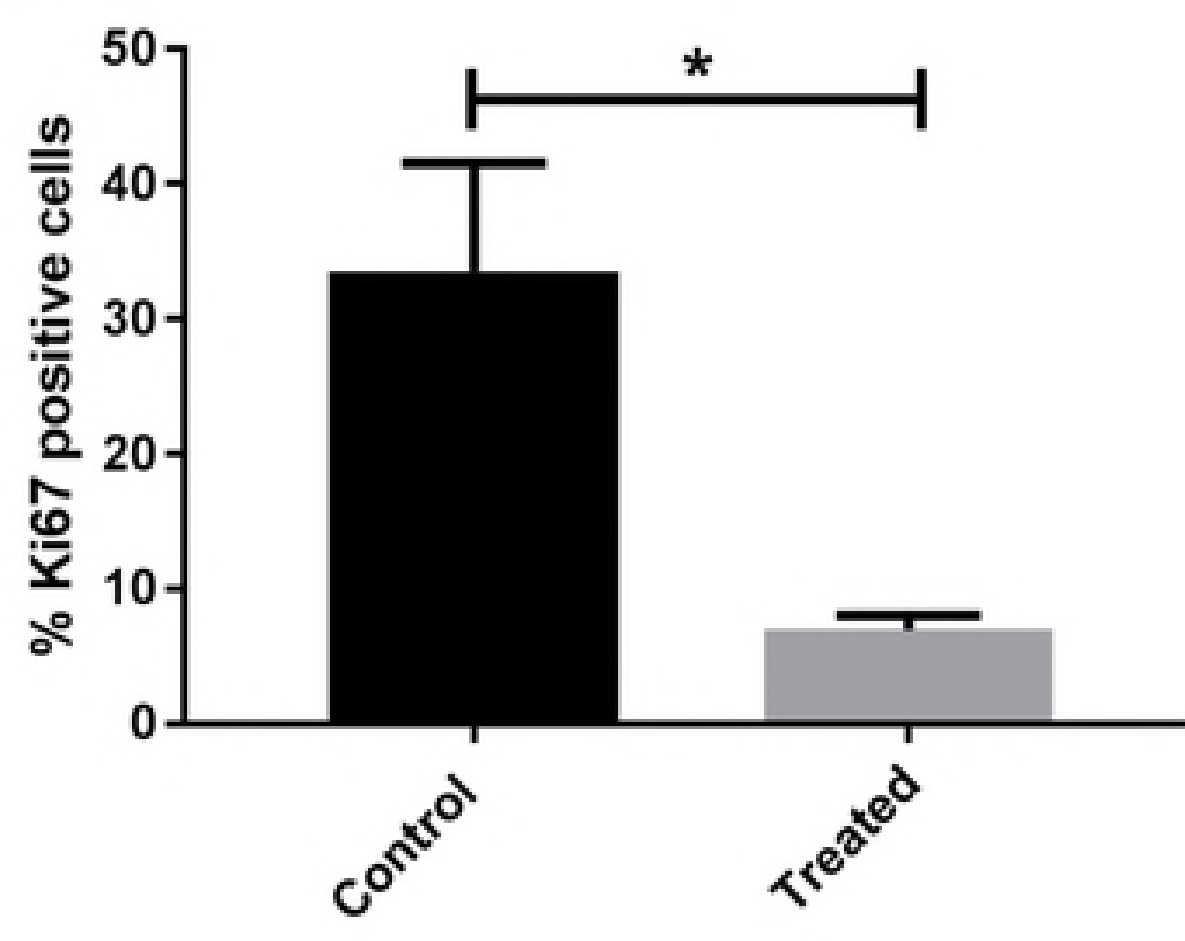

